\title{
Intermittent Auscultation
}

National Cancer Institute

\section{Source}

National Cancer Institute. Intermittent Auscultation. NCI Thesaurus. Code C114098.

A method of assessing intrapartum fetal heart rate during set intervals using an external device, such as a Doppler, fetoscope or stethoscope. 